\title{
Énoncé de position de l'ACMU sur le contrôle des armes à feu
}

\author{
Carolyn E. Snider, MD, MPH*; Howard Ovens, MD ${ }^{\dagger}$; Alan Drummond, MDCM ${ }^{\dagger}$; \\ Atul K. Kapur, MD, MSc ${ }^{\S}$
}

\begin{abstract}
Voir ARTICLE CONNEXe À LA PAGE 3
\end{abstract}
ENGLISH VERSION ON PAGE 64

\section{RÉSUMÉ}

Les blessures et les décès par armes à feu continuent d'être un problème important au Canada. Depuis les années 90, les médecins d'urgence du Canada ont milité en faveur du contrôle des armes à feu. Cet article actualise la position de I'Association canadienne des médecins d'urgence (ACMU) à l'égard du contrôle des armes à feu.

Même si la couverture des médias porte généralement sur les homicides, la majorité des décès par balle est en réalité le résultat de suicides. Moins de $40 \%$ des blessures par armes à feu sont infligées intentionnellement par une autre personne. Depuis la mise en application du Registre des armes à feu en 1995 au Canada, on a constaté une réduction importante des suicides par balle et des homicides par un conjoint. La proposition d'assouplir la législation sur les armes à feu au Canada aura des répercussions importantes sur les décès et les blessures par balle. Il faut plutôt élargir les programmes axés sur la prévention du suicide, de la violence conjugale et de la violence liée aux gangs de rue.

La majorité des blessures par armes à feu intentionnelles ou non intentionnelles impliquent une infraction aux règles d'entreposage ou de maniement sécuritaire de ces armes. Le potentiel de préjudice futur en raison de l'entreposage ou du maniement non sécuritaire des armes à feu ou d'actes de vengeance perpétrés par des gangs de rue corrobore notre position voulant que les établissements de soins de santé déclarent obligatoirement à la police les blessures par balle (BPB). Par ailleurs, il faut mettre en application un système de surveillance national pour appuyer la recherche et orienter les futures politiques publiques et la législation.

En tant que médecins d'urgence, nous devons plaider en faveur du contrôle des blessures. Toutes les blessures et tous les décès par balle sont évitables, et nous devons préconiser une stratégie multidimensionnelle afin de réduire au minimum ce risque pour nos patients.

\section{ÉNONCÉ DE POSITION DE L'ACMU}

L'Association canadienne des médecins d'urgence recommande les mesures suivantes :

1. Continuer d'appuyer les dispositions originales du projet de loi C-68 et de la Loi sur les armes à

* Du département de médecine d'urgence, hôpital St. Michael, Toronto (Ont.); chargée de cours, département de médecine, Université de Toronto, Toronto (Ont.); † directeur, centre d'urgence Schwartz/Reisman, hôpital Mount Sinai, Toronto (Ont.); professeur agrégé, département de médecine familiale et communautaire, Université de Toronto, Toronto (Ont.); ₹ professeur adjoint de clinique, départements de médecine familiale et de médecine d'urgence, Université d'Ottawa; Ottawa (Ont.); professeur adjoint de clinique, département de médecine familiale, Université Queen, Toronto (Ont.); §professeur adjoint, département de médecine d'urgence, Université d'Ottawa, Ottawa (Ont.); président, Physicians for a Smoke-Free Canada, Ottawa (Ont.); directeur (district 8), Association médicale de I'Ontario, Toronto (Ont.); urgentologue, L'Hôpital d'Ottawa, Ottawa (Ont.); clinicien chercheur, Institut de recherche en santé d'Ottawa, Ottawa (Ont.); chercheur, Unité de recherche sur le tabac de l'Ontario, Toronto (Ont.)

Présenté le 8 novembre 2008; accepté le 8 novembre 2008

CJEM 2009;11(1):73-83 
feu et s'opposer activement à toute tentative d'abroger le Registre national des armes à feu (y compris l'enregistrement des armes d'épaule).

2. Préconiser la mise en œuvre par le gouvernement d'un système de surveillance national des blessures et des décès par armes à feu.

3. Élargir les programmes axés sur la prévention du suicide, de la violence conjugale et de la violence liée aux gangs de rue.

4. Appuyer une législation exigeant la déclaration obligatoire par les établissements de soins de santé des blessures par balle, mais non des blessures par arme blanche ou autres blessures résultant d'un acte violent.

5. Continuer d'appuyer la recherche sur les blessures et les décès par balle afin d'orienter les futures politiques publiques et la législation.

Adopté par le conseil de direction de l'ACMU en octobre 2008

\section{Contexte}

Les armes à feu sont une cause importante de blessures et de décès. En 2004, 743 Canadiens ont été tués par balle (2,4 pour 100000 personnes $)^{1}$, et malgré le fait que les médias mettent l'accent sur la criminalité en milieu urbain, les suicides comptaient pour $76 \%$ de ces décès. Les blessures par balles (BPB) ont des répercussions importantes sur notre système de santé. Au cours de l'exercice financier 2001-2002, les BPB étaient à l'origine de 606 hospitalisations $^{2}$. Les blessures qui avaient été infligées intentionnellement par une autre personne représentaient $37 \%$ (224) de ces cas, les blessures non intentionnelles, $34 \%$ (205), et les tentatives de suicide, $20 \%$ (121). Bon nombre de victimes de BPB ne sont pas hospitalisées; leur congé est autorisé à l'urgence. Bien que les données nationales sur les visites à l'urgence ne soient pas disponibles, en 2004-2005, 624 personnes se sont rendues à l'urgence en Ontario en raison de blessures par armes à feu, et 199 ont été hospitalisées ${ }^{3}$. En plus de la morbidité et de la mortalité, le coût médical total (y compris les coûts directs de soins et la perte de productivité) imputable aux blessures par armes à feu au Canada en 1991 a été estimé à 6,6 milliards de $\$ 4$.

Au Canada, les médecins d'urgence ont milité activement pour réduire la morbidité et la mortalité liées aux armes à feu en préconisant le contrôle des armes à feu et la recherche sur les blessures infligées intentionnellement. C'est vers la fin des années 90 que l'on s'est penché sur le rôle des médecins d'urgence au Canada ${ }^{5}$. Les récentes controverses sur l'enregistrement des armes d'épaule et la déclaration obligatoire des BPB font qu'il est temps de mettre à jour l'énoncé de position de l'ACMU sur ces questions. Les auteurs ont fait une revue de la littérature pertinente et ont étudié les initiatives internationales et canadiennes de contrôle des armes à feu, ainsi que les initiatives passées et présentes des médecins d'urgence. Le conseil de direction de l'ACMU a examiné l'énoncé de position proposé et approuvé l'énoncé définitif en octobre 2008.

\section{La propriété des armes à feu au Canada}

Au Canada, près de 7 millions d'armes à feu sont enregistrées. En mars 2007, plus de 1,9 million de Canadiens détenaient des permis pour armes à feu et 1,6 million possédait au moins 1 arme à feu enregistrée ${ }^{6}$. Parmi les propriétaires d'armes à feu, $76 \%$ possèdent un fusil, $67 \%$, un fusil de chasse et $12 \%$, une arme de poing ${ }^{7}$. On entend par armes à feu sans restriction les armes d'épaule ordinaires (carabines et fusils de chasse). Les armes à feu à autorisation restreinte sont les armes de poing non prohibées, les carabines et les fusils de chasse semi-automatiques et les autres armes à feu désignées « à autorisation restreinte » par le Code criminel. Les armes à feu prohibées sont certaines armes de poing, les armes d'épaule à canon tronqué, les armes à feu entièrement automatiques et les autres armes à feu désignées " prohibées » par le Code criminel. Ce sont dans les zones rurales que l'on retrouve le plus fort pourcentage de détenteurs d'armes à feu. Au Canada, le taux de possession légale le plus élevé est au Yukon et dans les Territoires du Nord-Ouest, (32 \% des adultes possèdent 1 ou plusieurs armes à feu) et le plus faible est en Ontario $(9 \%)^{7}$. La majorité des propriétaires $(74 \%)$ utilisent leurs armes à feu pour la chasse, le tir à la cible ou le tir sportif $(30 \%)$ ou les collectionnent (17\%). Seulement $4 \%$ des détenteurs d'armes à feu en possèdent une pour protéger leurs biens ou pour leur protection personnelle.

Les 2 principales sources des armes à feu ayant entraîné la mort sont : 1) des armes à feu légales (c'est-à-dire celles qui sont enregistrées par un individu et en possession d'un individu qui détient un permis) qui sont utilisées à mauvais escient, le plus souvent lors d'homicides par un conjoint, de suicides et de blessures mortelles acciden- 
telles; 2) des armes à feu illégales qui ont été volées aux propriétaires légitimes du permis ou vendues par ces derniers ${ }^{8}$. On a estimé que $30 \%$ des armes à feu utilisées pour commettre des actes criminels à Toronto sont détournées de leur utilisation légitime. La plupart des autres armes illégales sont des armes de contrebande américaine?

\section{Blessures et décès causés par des armes à feu}

\section{Suicides}

Le suicide est la deuxième cause de décès au Canada chez les personnes âgées de 10 à 34 ans et la neuvième cause de décès, toutes causes confondues ${ }^{10}$. En 1998, les décès par balle représentaient $22,1 \%$ des suicides (26,2 \% étaient des hommes et $6,6 \%$, des femmes) ${ }^{11}$. La majorité des suicides ne sont pas prémédités, mais sont des actes impulsifs. Lorsqu'une arme à feu est utilisée, les tentatives de suicide sont particulièrement mortelles (taux de réussite de $96 \%$ ), comparativement à une surdose, où seulement $6,5 \%$ des tentatives sont réussies ${ }^{12}$.

Le $\mathrm{D}^{\mathrm{r}}$ Kellermann et ses collaborateurs ${ }^{13}$ ont montré que la possession d'une arme à feu à domicile augmente le risque de suicide par balle, avec un ratio d'incidence approché de 4,8 [intervalle de confiance (IC) à $95 \%$ de 2,7 à $8,5]$. Plus récemment, le $\mathrm{D}^{\mathrm{r}}$ Miller et ses collaborateurs ${ }^{14}$ ont examiné la corrélation, sur une période de 22 ans, entre les changements dans les taux de suicide et la possession d'armes à feu. Leur étude révèle que pour chaque baisse de $10 \%$ dans la possession d'armes à feu, le taux de suicide par balle a diminué de 4,2 \% (IC à $95 \%$ de 2,3 à $6,1 \%$ ) et le taux global de suicide a connu une baisse de $2,5 \%$ (IC à $95 \%$ de 1,4 à 3,6\%). Le rapport est plus grand pour les enfants (âgés de 0 à 19 ans). Ce groupe affichait une baisse du taux de suicide par balle de 8,3\% (IC à $95 \%$ de 6,1 à $10,5 \%$ ) et une réduction du taux global de suicide de $4,1 \%$ (IC à $95 \%$ de 2,3 à 5,9\%). Selon une étude sur les

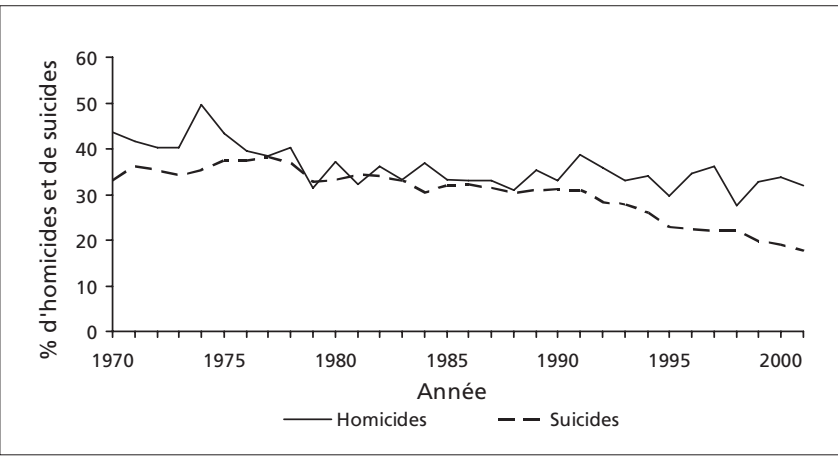

Fig. 1. Pourcentage de tous les homicides et les suicides commis avec arme à feu, 1970-2001. Adapté de Causes de décès, Statistique Canada ${ }^{18}$. blessures autoinfligées et les suicides par arme à feu chez les enfants (< 19 ans), $65 \%$ étaient causés par une arme à feu appartenant à un membre du ménage ${ }^{15}$. En outre, le taux de suicide est plus élevé dans les collectivités autochtones rurales, où il $\mathrm{y}$ a un plus grand usage des armes à feu ${ }^{16}$.

Le taux de suicide commis au moyen d'armes à feu chez les hommes au Canada a diminué entre 1979 et 1998, passant de 41 à $29 \%$ de tous les suicides ${ }^{11}$. Dans l'ensemble, les suicides par balle ont diminué de $43 \%$ depuis l'introduction de lois plus strictes sur les armes à feu en 1991 et de $23 \%$ depuis l'introduction de la Loi sur les armes à feu $1{ }^{17}$ en 1995 (Fig. $1^{18}$ ). Cette baisse a été confirmée dans une revue de 4 études qui examinait les changements dans les taux de suicide suite à l'introduction de lois plus rigoureuses sur les armes à feu en $1991^{19}$.

\section{Homicides}

\section{Vue d'ensemble}

Les homicides sont parmi les 10 principales causes de décès au Canada chez les personnes âgées de 1 à 34 ans ${ }^{10}$. En dépit de l'augmentation de la violence des gangs et des homicides commis à l'aide d'armes à feu illégales, les homicides par balle ont diminué sensiblement depuis l'introduction d'un contrôle plus strict des armes à feu en 1991, passant de 271 (0,99 pour 100000 habitants) en 1991 à $190(0,58$ pour 100000 habitants $)$ en $2006^{20}$.

Ceux qui s'opposent à l'enregistrement des armes d'épaule prétendent que peu d'actes criminels sont commis avec de telles armes ${ }^{21}$. Ce n'est pourtant pas le cas (Fig. 2). Des homicides commis au moyen d'une arme à feu en 2005 au Canada, $25 \%$ avaient été perpétrés avec des

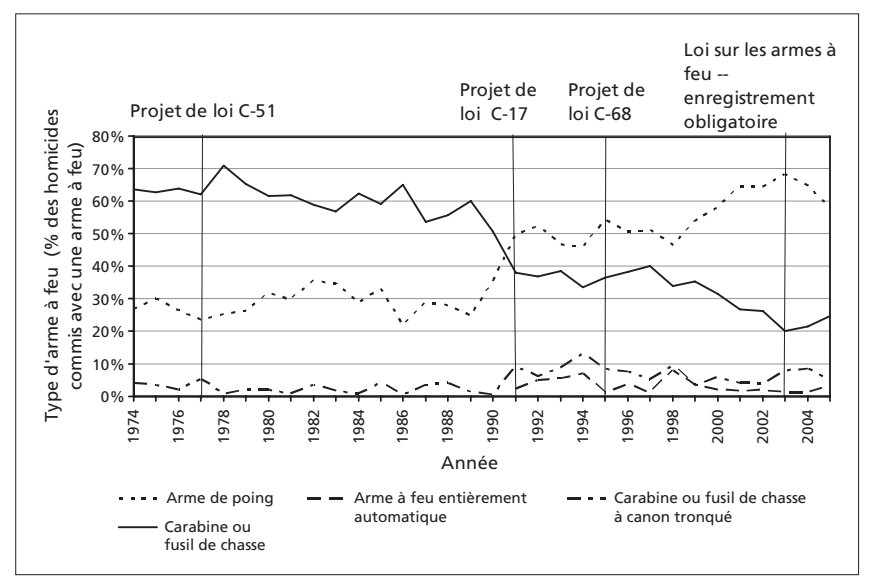

Fig. 2. Homicides commis au Canada avec une arme à feu, selon le type d'arme à feu de 1974 à 2005. Adapté du tableau 253-0005, Enquête sur les homicides, homicides commis avec une arme à feu, selon le type d'arme à feu, Canada. Centre canadien de la statistique juridique ${ }^{22}$. 
carabines ou des fusils de chasse, $58 \%$ avec des armes de poing et $18 \%$ avec des armes à feu prohibées ${ }^{22}$. Les armes d'épaule ont été utilisées dans $72 \%$ des homicides conjugaux $^{23}$. En outre, 10 des 13 agents de police tués dans l'exercice de leurs fonctions au cours de la dernière décennie ont été assassinés par des armes d'épaule $(77 \%)^{24}$. Depuis la mise en œuvre du Registre des armes à feu en 1995, le nombre d'homicides commis à l'aide d'armes d'épaule a chuté de $30 \%{ }^{22}$.

On note également certaines différences entre les zones urbaines et rurales. Les armes d'épaule sont utilisées plus souvent dans les crimes commis en régions rurales, tandis que les armes de poing sont utilisées dans la plupart des crimes en milieu urbain, conclut une étude nationale sur les armes à feu illégales8. À Toronto, entre 1998 et 2003, une arme de poing avait été utilisée dans $93 \%$ des homicides avec arme à feu ${ }^{25}$.

En 2006, le service de police a récupéré 61 (36\%) armes à feu qui avaient été utilisées dans des homicides ${ }^{26}$. De ce nombre, 18 (30\%) étaient enregistrées (12 carabines ou fusils de chasse, 4 armes de poing et 2 carabines ou fusils de chasse à canon tronqué). Les services de police ont été en mesure d'établir l'identité du propriétaire dans 45 cas (74\%) : 26 armes appartenaient à l'auteur présumé, 2 à la victime et 17 autres appartenaient à une personne autre que l'auteur présumé ou la victime (10 de ces 17 avaient été rapportées volées).

\section{Homicides entre conjoints}

Il existe des variations significatives entre les homicides conjugaux par balle et les autres homicides par balle, quant au type d'armes à feu en cause et aux facteurs de risque. En 2006, $21 \%$ des homicides étaient des homicides entre conjoints $(0,28 \text { pour } 100000)^{27}$ (Fig. 3). Depuis l'adoption de lois plus strictes sur le contrôle des armes à feu, le nombre d'homicides conjugaux à l'aide d'une arme à feu a diminué de $36 \%{ }^{23}$.

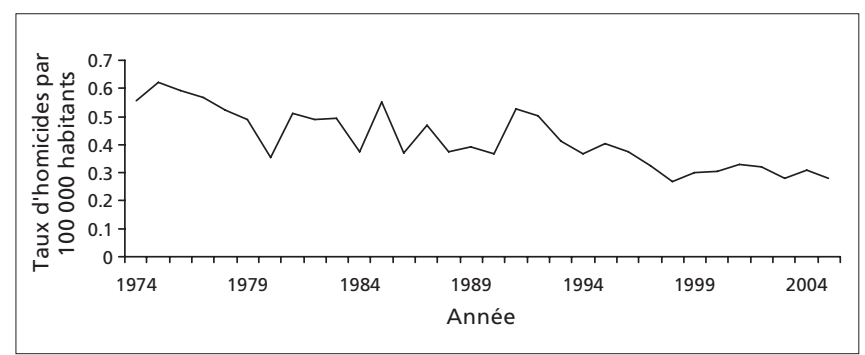

Fig. 3. Taux d'homicides pour 100000 habitants au Canada, de 1974 à 2005. Adapté du tableau 253-0006, Enquête sur les homicides, nombre d'homicides résolus, selon le type de relation entre l'accusé et la victime, Canada. Centre canadien de la statistique juridique ${ }^{27}$.
Le taux d'homicides conjugaux perpétrés contre les femmes est 5 fois plus élevé que contre les hommes ${ }^{28}$. Entre 1995 et 2004, les armes à feu étaient en cause dans $31 \%$ des homicides de conjointes ${ }^{23}$. Les carabines et les fusils de chasse ont été utilisés dans $62 \%$ des homicides conjugaux, les armes de poing, dans $28 \%$, et les fusils de chasse ou les carabines à canon tronqué, dans $10 \%$ de ces homicides.

La présence d'une arme à feu dans un domicile augmente le risque d'homicide entre conjoints ${ }^{29}$. Qui plus est, les armes à feu ne sont pas utilisées uniquement pour commettre des homicides conjugaux. Des propriétaires d'armes à feu participant à un programme d'intervention pour conjoints violents au Massachusetts avouent avoir intimidé leurs conjoints ainsi : 1) menacer de les abattre ou de tuer par balle un animal de compagnie ou une personne qui leur est chère; 2) nettoyer, tenir ou charger une arme à feu pendant un argument; ou 3) tirer une arme à feu pendant un argument $^{30}$. Depuis l'adoption de lois américaines interdisant la possession d'armes à feu par les personnes visées par une injonction restrictive en raison de violence familiale, le taux d'homicide d'un conjoint a diminué de $7 \%{ }^{31}$.

Au Canada, lorsqu'une personne fait une demande de permis de possession d'armes à feu, elle doit en informer ses partenaires conjugaux actuels et antérieurs. De plus, les conjoints peuvent appeler un service téléphonique 24 heures par jour s'ils ont des inquiétudes concernant leur sécurité. Plus de 26000 appels ont été faits à cette ligne téléphonique depuis son introduction en $1998^{32}$. Lorsque les agents de police répondent à un appel de violence familiale, ils doivent retirer les armes à feu légales du domicile et demander aux résidents s'il y a d'autres armes à feu non enregistrées ${ }^{33}$. Avant de répondre à un appel, les agents de police peuvent interroger le Registre des armes à feu afin de déterminer la présence d'armes à feu dans le domicile. En moyenne, les agents interrogent le registre 6300 fois par jour'.

\section{Homicides liés aux gangs}

La violence des gangs et des jeunes et la question des blessures et des décès par armes à feu sont des sujets qui se chevauchent de près. Les jeunes qui font partie de gangs de rue demeurent souvent dans des gangs à l'âge adulte.

Bien que les taux globaux d'homicides aient diminué considérablement depuis le début des années 90 , le taux de jeunes accusés d'homicides au Canada a atteint, en 2006, un niveau sans précédent depuis $1961^{34}$. Au cours de cette année, 104 homicides liés aux gangs (incluant les jeunes et les adultes) ont été commis (17\% de tous les homicides). Environ $50 \%$ de ces homicides ont eu lieu à Montréal, à Toronto, à Edmonton et à Vancouver, et $75 \%$ ont été com- 
mis avec des armes à feu, la plupart avec des armes de poing $^{26}$. Même s'il faut adopter une stratégie multidimensionnelle pour réduire les homicides liés aux gangs, il faut aussi interdire l'accès aux armes à feu pour obtenir des résultats positifs.

\section{Blessures et morts non intentionnelles par balle}

Les morts non intentionnelles par balle représentent un faible pourcentage, bien qu'important, de l'ensemble des morts par armes à feu, soit $3 \%$ en $2004^{1}$. Bon nombre des victimes étaient des enfants; d'autres étaient des chasseurs et des tireurs sportifs. Dans la majorité des cas, les blessures non mortelles causées par une arme à feu sont accidentelles. En Ontario, $63 \%$ de toutes les visites à l'urgence en 2004-2005 pour raison de BPB ont été classées comme non intentionnelles; en tout, 395 visites à l'urgence et 78 hospitalisations étaient le résultat de BPB non intentionnelles ${ }^{3}$.

Dans une étude sur les blessures et les morts non intentionnelles par balle d'enfants et d'adolescents, l'arme utilisée était, dans $72 \%$ des cas, la propriété d'un membre du ménage, d'un membre de la famille, d'un ami ou d'un parent d'un ami de la victime ${ }^{15}$.

On a proposé plusieurs interventions pour réduire les blessures et les morts non intentionnelles par arme à feu. Il a été prouvé que la conformité aux règles d'entreposage sécuritaire protège les enfants et les adolescents contre les blessures involontaires et les suicides ${ }^{35}$. Ces mesures sont prévues par la Loi sur les armes à feu ${ }^{36}$. Des lois sur l'entreposage sécuritaire dans certains États américains ont réduit la mortalité infantile causée par les armes à feu ${ }^{37}$. La sensibilisation aux armes à feu et la distribution de coffresforts pour armes à feu et de verrous d'armes sont des mesures qui ont connu du succès dans la promotion de l'entreposage sécuritaire des armes à feu ${ }^{38}$.

De nombreux groupes d'armes à feu, y compris la National Firearms Association du Canada, suggèrent une stratégie "protège-enfants », qui préconise l'éducation des enfants sur la sécurité des armes à feu ${ }^{39}$. On a étudié en détail des programmes de formation adaptés à l'âge offerts aux États-Unis. Dans le cadre d'un essai randomisé, on a fait subir un test avant et après la formation à des enfants. L'essai conclut que les enfants d'âge scolaire ont retenu l'information qu'ils ont apprise $e^{40}$, mais l'essai n'a pas examiné l'impact de la formation dans la vie réelle. Une autre étude a observé le comportement de garçons de 8 à 12 ans dans un scénario réaliste : les participants étaient placés dans une pièce avec un ami, un frère ou une sœur, ou les deux. On a placé dans la pièce une arme de poing et 2 pistolets à eau. Parmi ceux qui ont découvert l'arme à poing (72\%), $76 \%$ l'ont maniée, $48 \%$ ont appuyé sur la gâchette avec assez de force pour la déclencher, et environ $50 \%$ n'étaient pas certains si c'était une vraie arme à feu ou un jouet. Parmi ceux qui ont manipulé l'arme à feu ou appuyé sur la gâchette, $90 \%$ avaient été sensibilisés aux armes à feu ${ }^{41}$. Il est évident que l'éducation sur la sécurité des armes à feu n'est pas suffisante en soi et qu'elle ne peut être la seule mesure employée pour assurer la sécurité de nos enfants. Il est impératif que nous veillions à ce que les enfants n'aient pas accès aux armes à feu.

Les chasseurs et les tireurs sportifs sont également à risque de blessures accidentelles. Un examen de morts dues à la chasse en Caroline du Nord entre 1983 et 1992 a révélé que la victime a été prise pour du gibier dans $22 \%$ des cas. L'adoption de la loi Hunter Orange en 1988 voulant que les chasseurs portent des vêtements orange vif a permis de réduire de façon statistiquement significative le nombre de morts liées à la chasse ${ }^{42}$. Les propriétaires d'armes à feu sont également à risque de blessures lorsqu'ils nettoient ou transportent leurs armes à feu. Depuis 1991, la délivrance d'un permis à un particulier est subordonnée à la réussite d'un cours sur la sécurité des armes à feu ${ }^{43}$.

M. Vernick et ses collaborateurs ${ }^{44}$ ont suggéré que l'adoption de modifications à la conception des armes à feu telles que la création d'armes personnalisées (c'est-àdire des armes à feu avec lecteur d'un NIP ou d'empreintes digitales qui ne peuvent être utilisées que par l'utilisateur autorisé), l'utilisation d'indicateurs de chargement et de dispositifs de sécurité du chargeur pourraient diminuer considérablement le nombre de morts accidentelles par balle et réduire les dangers liés aux armes à feu volées.

\section{Armes à feu illégales}

La plupart des armes à feu utilisées lors d'homicides par des gangs et des jeunes sont illégales. Ce sont des armes de contrebande américaines ou des armes volées d'un propriétaire légitime canadien. L'acquisition illégale d'armes à feu des États-Unis a 5 grandes sources : 1) les revendeurs américains autorisés; 2) les prête-noms (qui se procurent légalement une arme à feu pour une personne incapable d'acheter légalement des armes); 3) la fausse identité : de fausses pièces d'identité utilisées par quelqu'un incapable d'acheter légalement des armes; 4) les marchés secondaires américains comme les expositions d'armes à feu, les marchés aux puces et les ventes privées; et 5) les vols. Lorsque le Service de police de Toronto a examiné les armes à feu impliquées dans des crimes à partir de 2003, $24 \%$ des armes à feu identifiables provenaient des États-Unis ${ }^{25}$.

Les armes à feu de résidence utilisées pour commettre 
des crimes de gangs sont le plus souvent volées de propriétaires légitimes. Depuis 1974, plus de 85000 armes à feu ont été volées, dont plus de $50 \%$ sont des armes à feu à autorisation restreinte (p. ex., les armes de poing) ${ }^{8}$. Entre 1998 et 2002, seulement $34 \%$ des armes à feu disparues ou volées ont été retrouvées ${ }^{45}$. Par conséquent, de nombreuses armes à feu volées demeurent dans les mains de criminels et sont utilisées dans des homicides attribuables à des gangs. Il est primordial d'exiger un entreposage sécuritaire et d'enregistrer les armes à feu pour pouvoir retracer celles qui sont volées.

\section{Historique du contrôle des armes à feu au Canada}

Le Canada a maintenu un strict contrôle des armes à feu depuis plus d'un siècle ${ }^{43}$. Le premier Code criminel, adopté en 1892, exigeait que les citoyens aient un permis pour porter un pistolet. Au milieu des années 30, les lois sur les armes à feu ont été renforcées; il est dorénavant obligatoire d'enregistrer les armes de poing. Au cours des 40 prochaines années, la portée des dispositions du Code criminel est élargie pour restreindre la possession d'armes automatiques.

Un changement majeur dans le contrôle des armes s'est produit en 1977 lorsque le projet de loi C-51 a été adopté. Il exige une autorisation d'acquisition d'armes à feu (AAAF). Pour ce faire, tous les candidats doivent faire l'objet d'un dépistage. Le projet de loi C-51 a également introduit des peines minimales obligatoires pour les actes criminels commis avec une arme à feu, la prohibition de toutes les armes entièrement automatiques et l'interdiction du port d'une arme à feu par les particuliers aux fins de protection de leurs biens.

En 1991, le projet de loi C-17 a renforcé les exigences de l'AAAF. On exige des vérifications plus approfondies sur les demandeurs, l'obligation de suivre une formation sur la sécurité et une période d'attente obligatoire de 28 jours.

En 1995, le projet de loi C-68 établit la Loi sur les armes à feu, qui sépare du Code criminel les aspects administratifs et réglementaires de la gestion des armes à feu. Parmi les principales modifications apportées, citons l'établissement de peines plus sévères pour les personnes reconnues coupables d'utiliser des armes à feu dans des crimes graves (un meurtre par exemple), l'enregistrement obligatoire de toutes les armes à feu; le contrôle de la vente de munitions; des dispositions d'entreposage plus strictes; la notification obligatoire du conjoint d'un demandeur de nouveau permis ou de renouvellement de permis ou d'enregistrement d'armes à feu; et l'interdiction d'armes militaires semi- automatiques et d'armes de poing à canon court. Le projet de loi C-68 a été fortement contesté par des opposants au contrôle des armes à feu (y compris la province de l'Alberta), qui prétendaient qu'il était inconstitutionnel. La Cour suprême du Canada a toutefois confirmé sa constitutionnalité en 1999. Toutes les armes à feu au Canada devaient être enregistrées au 1er janvier 2003. La mise en application du Registre des armes à feu a été entravée par la non-conformité active des opposants de la loi, mais la Gendarmerie royale du Canada a estimé qu'en mars 2007, $90 \%$ des quelque 2 millions de Canadiens propriétaires d'armes à feu avaient enregistré leurs armes ${ }^{6}$.

En février 2006, le Parti conservateur du Canada a formé un gouvernement minoritaire après avoir mené une campagne qui favorisait le retrait du Registre de certains types d'armes, essentiellement des armes de chasse et l'investissement de l'argent économisé dans des mesures de lutte contre la criminalité. Peu de temps après avoir prêté serment, les conservateurs ont présenté le projet de loi C-21, qui visait à supprimer l'obligation d'obtenir un certificat d'enregistrement pour les armes à feu sans restrictions, comme les carabines et les fusils de chasse. Les autres partis de la Chambre des communes, qui appuient tous le Registre, se sont opposés à ce projet de loi, mais le gouvernement a trouvé un autre moyen de l'immobiliser. En mai 2006, le gouvernement a introduit une « amnistie » d'un an pour les propriétaires d'armes de chasse qui n'ont pas renouvelé leur permis ou enregistré leurs armes d'épaule. Il l'a depuis prorogé à deux reprises. L'amnistie est en vigueur jusqu'en mai $2009^{46,47}$. Le projet de loi C-24, qui a été introduit en octobre 2007, modifierait le Code criminel et la Loi sur les armes à feu pour supprimer l'obligation d'obtenir un certificat d'enregistrement à l'égard des armes à feu sans restrictions ${ }^{48}$.

\section{Contrôle international des armes à feu}

Environ 200000 décès par armes à feu utilisées dans les suicides, les homicides et les blessures non intentionnelles surviennent dans le monde en dehors de contextes de guerre. En 1997, le Center for Disease Control des ÉtatsUnis a indiqué que les enfants de moins de 15 ans étaient 9 fois plus à risque de mourir de blessures par balle non intentionnelles, 11 fois plus susceptibles de se suicider avec une arme à feu et 16 fois plus susceptibles d'être victimes d'un homicide commis avec une arme à feu que les enfants dans 26 autres pays industrialisés ${ }^{49}$. En 2004, un sondage national sur les armes à feu réalisé aux États-Unis révélait que $38 \%$ des ménages et $26 \%$ des particuliers ont déclaré posséder au moins 1 arme à feu ${ }^{50}$. Près de $22 \%$ 
des ménages canadiens possèdent une arme à feu ${ }^{51}$. Les plus récentes statistiques en provenance du Canada et des États-Unis (2004) montrent que le taux d'homicides par arme à feu aux États-Unis est 7,2 fois plus élevé qu'au Canada; le taux de vols qualifiés impliquant des armes à feu aux États-Unis est 4,7 fois plus élevé qu'au Canada ${ }^{52}$. La réglementation américaine sur les armes à feu est extrêmement controversée. La plupart des lois sur les armes à feu sont instaurées au niveau de l'État, créant une grande divergence d'un État à l'autre et rendant difficile l'application des règlements. De nombreux groupes en faveur des armes à feu (y compris la National Rifle Association) font constamment pression sur le gouvernement des États-Unis, soutenant que la possession d'armes à feu est un droit constitutionnel et que par conséquent aucune restriction ne devrait être appliquée ${ }^{53}$. Les groupes en faveur du contrôle des armes à feu, comme la Campagne Brady, exercent des pressions sur le gouvernement pour accroître le contrôle des armes à feu. La coalition Mayors Against Illegal Guns, qui regroupe plus de 270 maires, est une autre initiative américaine qui vise à réduire le nombre de décès par balle. Les membres de la coalition partagent un objectif commun : éliminer les armes illégales de la rue ${ }^{54}$.

La réglementation sur le contrôle des armes à feu a eu un effet positif dans de nombreux pays. L'Australie a interdit les armes à feu semi-automatiques et les fusils à pompe en 1996, à la suite d'un massacre impliquant des armes à feu en Tasmanie. Cette mesure à eu pour résultat une décennie sans tuerie et un déclin accéléré des homicides et des suicides par armes à feu ${ }^{55}$. En 1989, l'Angleterre et le pays de Galles ont adopté une législation nationale sur la propriété d'armes à feu, l'enregistrement et l'entreposage. On y a observé une réduction significative des suicides par armes à feu après que ce projet de loi a été adoptés ${ }^{56}$.

\section{Historique des initiatives de médecins d'urgence au Canada}

\section{Participation au débat des années 90 sur le contrôle des armes à feu}

L'ACMU prend part au débat sur le contrôle des armes à feu depuis le début des années 90. Elle est devenue membre de la Coalition pour le contrôle des armes en 1992 et, entre 1994 et 1995, elle a rédigé son premier énoncé de position sur le contrôle des armes à feu. Elle recommandait notamment : 1) d'appuyer la création d'un registre national des armes à feu; 2) d'appuyer pleinement le projet de loi C-68; 3) d'envisager le développement d'un système de déclaration pour les personnes susceptibles d'être victimes de BPB (c'est-à-dire, les cas de dépression non traitée, de psychose, de toxicomanie et d'alcoolisme et de troubles du contrôle des impulsions) et celles impliquées dans des actes de violence familiale; 4) de lancer un appel en faveur d'un effort concerté pour élaborer et mettre en place des programmes d'éducation sur la violence et la résolution de conflits 5 .

Cette position a été présentée devant le Comité permanent de la justice et des affaires juridiques de la Chambre des communes en mai 1995 et le Comité sénatorial des affaires juridiques et constitutionnelles en septembre 1995.

Lors de l'assemblée annuelle de 2006, les membres ont enjoint aux membres du conseil de direction de l'ACMU de mettre à jour l'énoncé de position sur le contrôle des armes à feu en raison de la campagne de l'heure du gouvernement qui voulait supprimer l'obligation d'enregistrer les armes d'épaule. Le présent document est le résultat de cette demande.

\section{Déclaration obligatoire de blessure par balle au Canada}

La déclaration obligatoire des BPB par les fournisseurs de soins de santé et les établissements médicaux a fait l'objet de controverses au Canada. En 2005, l'Ontario a adopté le projet de loi 110, la première loi au Canada exigeant que les établissements de santé déclarent aux autorités légales le nom de toute personne se présentant avec une $\mathrm{BPB}^{57}$. En mars 2007, la Saskatchewan a adopté le projet de loi 20 : The Gunshot and Stab Wounds Mandatory Reporting Act (une loi sur la déclaration obligatoire des blessures par balle et par arme blanche $)^{58}$. En novembre 2007, la Nouvelle-Écosse a introduit une loi similaire, le projet de loi 10 : Gunshot and Stab Wounds Mandatory Reporting Act, mais après un débat public, elle a supprimé toute référence aux blessures par arme blanche avant d'adopter le projet de loi en décembre $2007^{59}$. Le Manitoba a adopté le projet de loi 20 : Loi sur la déclaration obligatoire des blessures par balle et par arme blanche en juin $2008^{60}$.

\section{L'expérience de l'Ontario}

En 2003, la section de la médecine d'urgence de l'Association médicale de l'Ontario (AMO) a publié un énoncé de position proposant l'adoption d'une loi sur la déclaration obligatoire de BPB61. Elle a fait valoir que chaque fois qu'un patient est volontairement ou involontairement blessé par une arme à feu, il y a violation de l'entreposage ou du maniement sécuritaire d'une arme. Le potentiel de préjudice futur, que ce soit à l'égard de la victime ou d'autres personnes, à leur domicile ou à proximité, en raison de l'entreposage inadéquat ou du maniement non sécuritaire 
d'une arme à feu ou d'actes de vengeance perpétrés par des gangs de rue, démontre qu'il s'agit bel et bien d'une question de santé publique. La section propose que les mêmes mesures de protection de la santé publique soient appliquées pour les personnes blessées par balle que celles pour les cas de suspicions de violence envers les enfants, de patients qui semblent inaptes à conduire et la longue liste de maladies transmissibles à déclaration obligatoire : des cas que les médecins d'urgence étaient déjà tenus de déclarer. La section de la médecine d'urgence de l'AMO a également précisé qu'elle n'appuyait pas la déclaration obligatoire des blessures par arme blanche. Les BPB sont, intrinsèquement, différentes des autres blessures résultant d'actes violents (y compris les lésions produites par armes blanches) en raison de leur caractère potentiellement mortel, y compris la mortalité à distance. Les armes à feu représentent un risque pour la santé publique et pour les personnes à proximité lorsque quelqu'un appuie sur la gâchette $^{62}$. La déclaration de blessures par arme blanche représenterait, pour les fournisseurs de soins de santé et les services de police, un énorme fardeau qui serait extrêmement disproportionné par rapport au mince avantage potentiel qu'elle comporterait pour la santé. La section de la médecine d'urgence de l'AMO a également proposé la déclaration obligatoire comme moyen d'éviter les conflits entre les médecins d'urgence et les policiers lorsque des patients avec des BPB se présentent à l'urgence. La section a réalisé un sondage qui a révélé que les pratiques des médecins d'urgence de l'Ontario variaient énormément ${ }^{63}$. En outre, de nombreux médecins d'urgence et policiers croyaient à tort que la déclaration obligatoire était déjà en place.

La déclaration a soulevé la controverse; elle a provoqué chez certains de vives dissensions. Les opposants se préoccupaient du fait que certains patients pourraient éviter de se faire soigner ou tarderaient à le faire. Bon nombre étaient préoccupés par l'aspect déontologique de la déclaration aux autorités ${ }^{64}$. Toutes les autres formes de déclaration obligatoire sont faites auprès d'organismes autres que ceux chargés de l'application de la loi (par exemple, la Société d'aide à l'enfance, le ministère des Transports ou le département de la santé publique) $)^{65}$. Les opposants ont également fait valoir que les médecins d'urgence avaient déjà l'" obligation de mise en garde » dans les cas où ils estimaient que le public était à risque ${ }^{64}$. En outre, ils ont fait remarquer qu'aucune recherche n'avait montré que la déclaration obligatoire des BPB réduirait le nombre de blessures ou de morts par balle. Malgré cela, le projet de loi 110 a été adopté sans opposition au printemps 2005.

En 2007, une enquête a été menée pour examiner l'efficacité de la législation ontarienne sur la déclaration obliga- toire des $\mathrm{BPB}$ et les enjeux connexes ${ }^{66}$. Les répondants membres de la section sur la médecine d'urgence de l'OMA, les représentants de corps policiers et les citoyens choisis au hasard étaient largement d'accord avec la loi et l'appuyaient. Le taux d'observation de la loi par les travailleurs de la santé était élevé. Huit des 47 agents de police ont rapporté avoir été personnellement impliqués dans une enquête lancée à la suite de la déclaration d'un incident en vertu de la loi, ce qui a résulté à entre 6 et 14 accusations ou confiscations d'armes à feu. Les médecins d'urgence ont signalé 6 incidents où les patients avaient attendu avant de se faire soigner ${ }^{66}$. Comme il n'y a pas de base de données, on n'est pas en mesure de juger l'effet des déclarations, mais des données non scientifique appuient les avantages potentiels (enquêtes menant à la confiscation d'armes à feu ou à des mises en accusation, ou les deux) et les préjudices potentiels (victimes qui tardent à se faire soigner afin d'éviter d'être interrogées par la police). Aucun élément de preuve n'a été publié au Canada ou aux États-Unis sur les avantages de la déclaration obligatoire. D'autres recherches permettront de mieux évaluer cette initiative et guideront l'établissement de la législation au Canada. Toutefois, l'expérience à ce jour ne justifie pas l'élargissement du champ d'application des lois sur la déclaration obligatoire pour inclure les blessures par arme blanche ou d'autres formes d'activité criminelle.

(À la lumière de la discussion ci-dessus, les auteurs de cet article n'étaient pas d'accord, à savoir s'ils devaient ou non recommander la diffusion d'un énoncé de position sur la déclaration obligatoire des BPB. Au final, les auteurs ont présenté les deux côtés de la médaille devant le conseil de direction de l'ACMU, et le conseil a décidé d'appuyer la déclaration obligatoire des BPB.)

\section{Nécessité de poursuivre la recherche et les activités de représentation}

En octobre 2007, le gouvernement canadien actuel a déposé le projet de loi C-24, qui vise à supprimer l'obligation d'obtenir un certificat d'enregistrement pour les armes d'épaule. Pourtant, depuis la mise en œuvre du Registre, il y a eu une réduction importante du nombre de décès par armes à feu.

Il est impératif d'accroître le financement de la recherche au Canada sur 1) les causes fondamentales de la violence à tous les niveaux (sociétal, communautaire et individuel); 2) la santé mentale; 3) les interventions possibles pour réduire les BPB accidentelles. Il est également évident qu'il faut mettre en place un système national de surveillance des blessures et des décès par armes à feu. Une 
telle base de données serait très utile pour réaliser des recherches sur la santé et les politiques au Canada.

En tant que médecins, nous devons préconiser le contrôle des blessures. Nous avons la possibilité de faire un geste significatif pour la santé des Canadiens et devons nous opposer à l'assouplissement des mesures de contrôle des armes à feu au Canada. Nous avons vu, dans nos salles d'urgence, un trop grand nombre de patients blessés ou tués par balle. Toutes les blessures et les morts causées par des armes à feu sont évitables. Nous devons donc appuyer une stratégie multidimensionnelle si nous voulons alléger le fardeau qui retombe sur nos patients. Nous sommes d'avis que les mesures présentées dans cet énoncé de position de l'ACMU contribueront à réaliser ces objectifs.

Conflits d'intérêts : aucun déclaré.

\section{Références bibliographiques}

1. Mortalité : liste sommaire des causes - 2004. Ottawa, Ontario : Statistique Canada; 2007. No 84F0209XIF au catalogue. Disponible sur: www.statcan.gc.ca/pub/84f0209x/84f0209 x2004000-fra.pdf (consulté le 27 novembre 2008).

2. Institut canadien d'information sur la santé. Registre national des traumatismes (RNT) Rapport des faits saillants sur les hospitalisations à la suite de blessures, 2002 à 2004. 2004. Ottawa, Ontario : Le registre; 2007. Disponible sur : http://secure.cihi.ca /cihiweb/dispPage.jsp?cw_page=AR_8_F (consulté le 27 novembre 2008).

3. Smartrisk. Firearm Injuries. Toronto, Ontario : Compass. 2006;3. Disponible sur : www.oninjuryresources.ca/downloads /Compass/2006/2006-11-OICompass-firearm.pdf (consulté le 27 novembre 2008).

4. Miller TR. Costs associated with gunshot wounds in Canada in 1991. CMAJ 1995;153:1261-8.

5. Fisher H, Drummond A. A call to arms : the emergency physician, international perspectives on firearm injury prevention and the Canadian gun control debate. J Emerg Med 1999;17 :529-37.

6. Faits en bref sur le Programme canadien des armes à feu mars 2007. Ottawa, Ontario : Gendarmerie royale du Canada; 2007. Disponible sur : www.cfc-cafc.gc.ca/media/program _statistics/default_f.asp (consulté le 27 novembre 2008).

7. Le point sur les armes à feu. Ottawa, Ontario : Centre canadien des armes à feu; 1998. Disponible sur : www.cfc-cafc.gc.ca/ pol-leg/res-eval/pamplets/pdfs/focus-fr.pdf (consulté le 27 novembre 2008).

8. Canada. Dossier spécial : Marché illicite des armes à feu au Canada 2007. Ottawa, Ontario : Criminal Intelligence Service Canada; 2008. Disponible sur : www.cisc.gc.ca/annual_reports /annual_report_2007/feature_focus_2007_f.html (consulté le 27 novembre 2008).
9. Ontario. Document d'information : Le plan en quatre volets du gouvernement McGuinty pour protéger les collectivités de l'Ontario contre la violence armée. Toronto $(\mathrm{ON})$ : Ministère du procureur général; 2008. Disponible sur : www.attorney general.jus.gov.on.ca/french/news/2008/20080527-gun-bg-fr.pdf (consulté le 27 novembre 2008).

10. Canada. Principales causes de décès et d'hospitalisation au Canada. Ottawa, Ontario : Agence de la Santé publique du Canada; 2004. Disponible sur : www.phac-aspc.gc.ca/publicat /lcd-pcd97/table1-fra.php (consulté le 27 novembre 2008).

11. Langlois S, Morrison P. Suicide death and attempts. Health Rep $2002 ; 13: 9-23$.

12. Shenassa ED, Catlin SN, Buka SL. Lethality of firearms relative to other suicide methods : a population based study. J Epidemiol Community Health 2003;57 :120-4.

13. Kellermann AL, Rivara FP, Somes G, et al. Suicide in the home in relation to gun ownership. N Engl J Med 1992;327 :467-72.

14. Miller M, Azrael D, Hepburn L, et al. The association between changes in household firearm ownership and rates of suicide in the United States, 1981-2002. Inj Prev 2006;12:178-82.

15. Grossman DC, Reay DT, Baker SA. Self-inflicted and unintentional firearm injuries among children and adolescents : the source of the firearm. Arch Pediatr Adolesc Med 1999;153:875-8.

16. Canada. Tendances relatives aux taux de mortalité des Premières nations. Ottawa, Ontario : Santé Canada; 1996.

17. Loi sur les armes à feu. 1995, ch. 39. F-11.6. Disponible sur : http://lois.justice.gc.ca/fr/ShowFullDoc/cs/F-11.6///fr (consulté le 28 novembre 2008 ).

18. Causes de décès. Ottawa, Ontario : Statistique Canada. No 84208-XIF au catalogue.

19. Leenaars AA, Moksony F, Lester D, et al. The impact of gun control (Bill C-51) on suicide in Canada. Death Stud 2003;27 :103-24.

20. Centre canadien de la statistique juridique. Enquête sur les homicides, principales méthodes utilisées pour commettre l'homicide, Canada. Tableau 253-0002. Ottawa, Ontario : Statistique Canada; 2008. No 85F0033MWF au catalogue.

21. Law-abiding Unregistered Firearms Association. Random firearms registry quick facts. Edmonton $(\mathrm{AB})$ : The Association; 2008. Disponible sur : www.lufa.ca/quickfacts.asp (consulté le 27 novembre 2008).

22. Centre canadien de la statistique juridique. Enquête sur les homicides, homicides commis avec une arme à feu, selon le type d'arme à feu, Canada. Table 253-0005. Ottawa, Ontario : Statistique Canada; 2008.

23. Centre canadien de la statistique juridique. La violence familiale au Canada : un profil statistique 2006. Ottawa, Ontario : Statistique Canada; 2006. No 85-224-XIF au catalogue. Disponible sur : www.statcan.gc.ca/pub/85-224-x/85-224-x2005000-fra.pdf (consulté le 27 novembre 2008).

24. Canadian Association of Chiefs of Polices. Canadian Police and 
Peace Officer's Memorial. Ottawa, Ontario : The Association; 2007. Disponible sur : www.cacp.ca/english/memoriam/english /default.htm (consulté le 27 novembre 2008).

25. Procès-verbal de la réunion du Toronto Police Services Board. Toronto, Ontario : The Board. 22 janvier 2007.

26. Homicides 2006. Ottawa, Ontario : Le Quotidien. Statistique Canada; 17 octobre 2007. Disponible sur : www.statcan.gc.ca /daily-quotidien/071017/dq071017b-fra.htm (consulté le 27 novembre 2008).

27. Centre canadien de la statistique juridique. Enquête sur les homicides, nombre d'homicides résolus, selon le type de relation entre l'accusé et la victime, Canada. Tableau 253-0006. Ottawa, Ontario : Statistique Canada; 2008.

28. Homicides 2005. Ottawa, Ontario : Le Quotidien. Statistique Canada; 8 novembre 2006. Disponible sur : www.statcan .gc.ca/daily-quotidien/061108/dq061108b-fra.htm (consulté le 27 novembre 2008 ).

29. Campbell JC, Webster D, Koziol-McLain J, et al. Risk factors for femicide in abusive relationships : results from a multisite case control study. Am J Public Health 2003;93 :1089-97.

30. Rothman EF, Hemenway D, Miller M, et al. Batterers' use of guns to threaten intimate partners. J Am Med Womens Assoc $2005 ; 60: 62-8$.

31. Vigdor ER, Mercy JA. Do laws restricting access to firearms by domestic violence offenders prevent intimate partner homicide? Eval Rev 2006;30 :313-46.

32. Canada. Programme canadien de contrôle des armes à feu : Interdire l'accès à des armes à feu aux personnes qui ne doivent pas en avoir. Ottawa, Ontario : Ministère de la Justice; 2003. Disponible sur : http://canada.justice.gc.ca/fra/nouv-news/cp-nr /2003/doc_30840.html (consulté le 27 novembre 2008).

33. Code criminel L.R.C. 1985, c. C-46, art. 117.04.

34. Juristat. Ottawa, Ontario : Statistique Canada; 2006. No 85-002XIF au catalogue. Disponible sur : www.statcan.gc.ca/pub/ 85-002-x/85-002-x2001010-fra.pdf (consulté le 27 novembre 2008).

35. Grossman DC, Mueller BA, Riedy C, et al. Gun storage practices and risk of youth suicide and unintentional firearm injuries. JAMA 2005;293:707-14.

36. Canada. Loi sur les armes à feu : Règlement sur l'entreposage, l'exposition et le transport des armes à feu et autres armes par des entreprises. Ottawa, Ontario : Ministère de la Justice; 2007. Disponible sur : http://laws.justice.gc.ca/fr/showtdm/cr/ DORS-98-210 (consulté le 27 novembre 2008).

37. Hepburn L, Azrael D, Miller M, et al. The effect of child access prevention laws on unintentional child firearm fatalities, 1979-2000. J Trauma 2006;61 :423-8.

38. Carbone PS, Clemens CJ, Ball TM. Effectiveness of gun-safety counseling and a gun lock giveaway in a Hispanic community. Arch Pediatr Adolesc Med 2005;159:1049-54.

39. National Firearm Association. Gunproofing your child.
Edmonton (AB) : The Association; 2007. Disponible sur : www.nfa.ca/publications/gunproof.pdf (consulté le 27 novembre 2008).

40. Howard PK. Evaluation of age-appropriate firearm safety interventions. Pediatr Emerg Care 2005;21 :473-9.

41. Jackman GA, Farah MM, Kellermann AL, et al. Seeing is believing : What do boys do when they find a real gun? Pediatrics 2001; $107: 1247-50$.

42. Cina SJ, Lariscy CD, McGown ST, et al. Firearm-related hunting fatalities in North Carolina : impact of the 'Hunter Orange' Law. South Med J 1996;89 :395-6.

43. Historique du contrôle des armes à feu au Canada jusqu'à la Loi sur les armes à feu inclusivement. 2004. Ottawa, Ontario : Gendarmerie royale du Canada; 2007. Disponible sur : www.cfe -cafc.gc.ca/pol-leg/hist/firearms_control_f.asp (consulté le 27 novembre 2008).

44. Vernick JS, O'Brien M, Hepburn LM, et al. Unintentional and undetermined firearm related deaths : a preventable death analysis for three safety devices. Inj Prev 2003;9 :307-11.

45. Le rapport sur l'administration de la Loi sur les armes à feu présenté au solliciteur général du Canada présenté par le directeur de l'enregistrement des armes à feu. Ottawa, Ontario : Gendarmerie royale du Canada; 2002. No JS99-1/2002 au catalogue.

46. Canada. Décret modifiant le Décret fixant une période d'amnistie (2006). Ottawa, Ontario : Gazette du Canada; 2007. Disponible sur : http://canadagazette.gc.ca/partI/2007 /20070407/html/regle3-f.html (consulté le 27 novembre 2008).

47. Canada. Décret modifiant le Décret fixant une période d'amnistie (2006). Ottawa, Ontario : Gazette du Canada; 2008. Disponible sur : http://gazetteducanada.gc.ca/partII /2008/20080528/html/sor147-f.html (consulté le 27 novembre 2008).

48. Projet de loi C-21 : Loi modifiant le Code Criminel et la Loi sur les armes à feu (non-enregistrement des armes à feu ni prohibées ni à autorisation restreinte, $2 \mathrm{e}$ session, $39 \mathrm{e}$ législature, Canada, 2007.

49. Centers for Disease Control and Prevention. Rates of homicide, suicide, and firearm-related death among children -26 industrialized countries. Atlanta (GA) : The Centre; 1997. Disponible sur : www.cdc.gov/mmwr/preview/mmwrhtml/00046149.htm (consulté le 27 novembre 2008)

50. Hepburn L, Miller M, Azrael D, et al. The US gun stock : results from the 2004 national firearms survey. Inj Prev 2007;13:15-9.

51. Block R. Les armes à feu au Canada et dans huit autres pays occidentaux : résultats choisis du sondage international de 1996 (auprès des victimes) de crime.1997. Ottawa, Ontario : Ministère de la Justice; 1997. Disponible sur : www.cfc-cafc .gc.ca/pol-leg/res-eval/publications/1997/pdfs/crime_fr.pdf (consulté le 27 novembre 2008).

52. Hung K. Firearm Statistics. Ottawa, Ontario : Ministère de la Justice; 2006. Disponible sur : www.justice.gc.ca/en/ps/rs/rep /2006/rr06-2/table.html (consulté le 27 novembre 2008). 
53. National Rifle Association. Disponible sur : www.nraila.org /about/

54. Mayors Against Illegal Guns Coalition. Disponible sur : http: //mayorsagainstillegalguns.org/html/about/about.shtml

55. Chapman S, Alpers P, Agho K, et al. Australia's 1996 gun law reforms : faster falls in firearm deaths, firearm suicides, and a decade without mass shootings. Inj Prev 2006;12 :365-72.

56. Hawton K, Fagg J, Simkin S, et al. Methods used for suicide by farmers in England and Wales. The contribution of availability and its relevance to prevention. Br J Psychiatry 1998;173:320-4.

57. Projet de loi 110, Loi exigeant la divulgation à la police de renseignements en ce qui concerne les personnes traitées pour blessure par balle, 1re session, 38e législature, Ontario, 2005. Disponible sur : www.ontla.on.ca/bills/bills-files/38_Parliament /Session1/b110ra.pdf (consulté le 27 novembre 2008).

58. Saskatchewan. Progress of Bills 2006/07. Regina (SK) : Legislative Assembly of Saskatchewan; 2007. Disponible sur : www.legassembly.sk.ca/bills/ProgressOfBills/Progress_of_Bills _2006-2007.pdf (consulté le 27 novembre 2008).

59. Projet de loi 10, Gunshot and Stab Wounds Mandatory Reporting Act, 2e session, 60e législature, Nouvelle-Écosse, 2007. Disponible sur : www.gov.ns.ca/legislature/legc/bills/60th _2nd/1st_read/b010.htm (consulté le 27 novembre 2008).

60. Projet de loi 20, Loi sur la déclaration obligatoire des blessures par balle et par arme blanche. 2008, 2e session, 39e législature, Manitoba, 2008. Disponible sur : http://web2.gov.mb.ca/laws /statutes/2008/c02108f.php (consulté le 27 novembre 2008).

61. Ontario Medical Association. The case for mandatory reporting of gunshot wounds in the emergency department. Toronto,
Ontario : The Association; 2003. Disponible sur : www.oma.org /pcomm/OMR/nov/03gunshot.htm (consulté le 27 novembre 2008).

62. Ovens H, LeBlanc B. OMA submission to the Standing Committee on Justice Policy on Bill 110 : an act to require the disclosure of information to police respecting persons being treated for gunshot wounds. Toronto, Ontario : The Association; 2005. Disponible sur : www.oma.org/pcomm/OMR/apr/05bill110.htm (consulté le 27 novembre 2008).

63. Ovens H, Morrison H, Drummond A, et al. The case for mandatory reporting of gunshot wounds in the emergency department. Toronto, Ontario. The Ontario Medical Association; 2003. Disponible sur : www.oma.org/pcomm/OMR/nov/03gunshot.htm (consulté le 27 novembre 2008).

64. Pauls MA, Downie J. Shooting ourselves in the foot : why mandatory reporting of gunshot wounds is a bad idea. CMAJ $2004 ; 170: 1255-6$.

65. The Standing Committee on Justice Policy. Deliberations of Bill 110 - Mandatory Gun-shot Wounds Reporting Act. 2005. Toronto, Ontario : Legislative Assembly of Ontario; 2005. Disponible sur : www.ontla.on.ca/committee-proceedings /transcripts/files_html/2005-03-02_JP022.htm (consulté le 27 novembre 2008).

66. Ovens H, Borgundvaag B, Park H. Reaction in Ontario to Bill 110 : Canada's first mandatory gunshot wound reporting law. CJEM 2009;11:3-13.

Correspondance : $\mathrm{D}^{\text {re }}$ Carolyn Snider, département de médecine d'urgence, hôpital St. Michael, 30, rue Bond, Toronto ON M5B 1W8; sniderc@smh.toronto.on.ca 